Proc. Indian Acad. Sci., Vol. 87 A (Mathematical Seiences-2), No. 5, May 1978, pp. 85-105, (C) printed in India.

\title{
Phase space methods and the Hamilton-Jacobi form of dynamics
}

\author{
N MUKUNDA \\ Centre for Theoretical Studies, Indian Institute of Science, Bangalore 560012 \\ MS received 24 February 1978
}

\begin{abstract}
A general analysis of the Hamilton-Jacobi form of dynamics motivated by phase space methods and classical transformation theory is presented. The connection between constants of motion, symmetries, and the Hamilton-Jacobi equation is described.
\end{abstract}

Keywords. Classical dynamics; phase-space method; Hamilton-Jacobi theory.

\section{Introduction}

The formulation of classical dynamics based on the Hamilton-Jacobi (H-J) equation has played an important role in the development of quantum mechanics. In the days of the old quantum theory it was used most effectively by Sommerfeld, Wilson, Epstein and Einstein among others to extend the Bohr quantization condition, originally formulated for the hydrogen atom, to more complex systems (Sommerfeld [14]; Wilson [19]; Epstein [6]; Einstein [5]; see also Born [1], Sommerfeld [15]. Later on Schrödinger [13] used the H-J equation as the starting point in his construction of wave mechanics based on de Broglie's theory of phase waves. Conversely, once the Schrödinger wave equation had been established, the H-J equation was recovered as the first step in solving the wave equation in the quasi-classical WKB approximation (Wentzel [17]; Kramers [7]; Brillouin [2]).

$i$ After the advent of quantum mechanics the study of invariance and symmetry properties of dynamical systems took on great importance. The linear structure of quantum mechanics expressed by the superposition principle gave to this study great elegance and simplicity, and enabled one to draw extensively upon the mathematical theory of group representations. At least partly due to the fact that for some time now quantum mechanics has been much more thoroughly studied and taught than classical mechanics, there is the feeling that the mathematical description of symmetry is easier to grasp in the quantum context than in the classical one. However it must be admitted that the mathematical description of symmetry is an important part of the formalism of classical mechanics. Classical descriptions of symmetry and invariance of course do exist, both in the Lagrangian form of mechanics and in the Hamiltonian phase space form. The intimate connection between symmetries and conservation laws expressed by the Noether theorems is an important aspect of the Lagrangian formulation (Noether [9]). And the realisation of symmetry operations as canonical transformations on phase space is an important aspect of the Hamiltonian formulation. 
Given all this it would seem somewhat surprising that there appears to be no easily accessible discussion of the $\mathrm{H}-\mathrm{J}$ form of dynamics which analyses the action of symmetry operations directly on the H-J equation and its solutions. (Several classic treatments of the H-J form of dynamics are, of course, available; see, for instance, Nordheim and Fues [10] Whittaker [18]; Lanczos [8]). The corresponding quantum discussion in which symmetry operations are implemented by the action of unitary transformations on solutions to the Schrödinger equation is of course well known. But a discussion of how concepts and methods natural to the phase space description of dynamics can be adapted to the $\mathrm{H}-\mathrm{J}$ description seems unavailable. The concept of the Poisson Bracket (PB) among phase space functions, and the generation of canonical transformations by given phase space functions which may in particular be constants of motion, are among the important elements of the phase space description of dynamics; and it would be instructive to see how these are related to the $\mathrm{H}-\mathrm{J}$ equation and its solutions. There is also another point of view from which it is illuminating to study the $\mathrm{H}-\mathrm{J}$ theory, which is the following. It is well known that an individual solution of the H-J equation describes a family of solutions of the Hamiltonian equations of motion, constructed in a special way. It is these classical families that stand in correspondence with individual solutions of the Schrodinger equation, which of course describe single states of motion in quantum mechanics. At the kinematic level a single function on configuration space (which in a well known way generates one of the above mentioned classical families of trajectories) is analogous to one Schrödinger wavefunction or more generally one state in quantum mechanics. For state vectors many characteristically quantum mechanical properties obtain. For instance, a state vector can be a simultaneous eigenvector of two distinct operators only if it is also an eigenvector of their commutator corresponding to the eigenvalue zero. Again a given state vector can always be expanded as a linear combination of, say, energy eigenstates, and there is a straightforward way to isolate individual terms in this expansion. All these features of quantum mechanics turn out to possess analogues in the H-J form of classical mechanics, some being better known than others. The purpose of this paper is to provide a study of the H-J theory from these points of view.

The H-J equation forms an important component of a highly developed part of classical mathematics having deep connections with the calculus of variations and with systems of ordinary differential equations (Caratheodory [3]; Rund [11]). However a discussion of it along the lines mentioned above seems unavailable.

The material of this paper is arranged as follows. In section 1, some useful geometrical ideas in phase space are introduced. The essential point is to associate a surface in phase space with every configuration space function in a special way and with certain intrinsic canonical invariant features. The action of canonical transformations on these surfaces and evaluation of PB's on them are analysed, permitting a simple discussion of simultaneous $\mathrm{H}-\mathrm{J}$ equations. Section 2 gives a detailed geometrical interpretation of solutions of the time-dependent $\mathrm{H}-\mathrm{J}$ equation, and the action of symmetry transformations on the manifold of solutions. The contrast between values of a dynamical variable and the transformations it generates is clearly exhibited. In section 3 the relations between solutions of the time dependent and time-independent $\mathrm{H}-\mathrm{J}$ equations is analysed motivated by similar situations in quantum mechanics. One is thereby led to a better understanding of a well known method of passing from one equation to the other. Section 4 introduces very 
briefly the notion of H-J equations on a Lie group. Section 5 contains concluding remarks. The appendix summarises some useful properties of canonical transformations and Lagrange brackets in phase space, and the general mathematical techniques for building up solutions of the H-J equations.

\section{Geometry of phase space surfaces}

The usual geometrical pictures that are constructed to aid in the understanding of the H-J equation are confined to configuration space. They are based on Huyghen's principle in optics and the analogy between optics and mechanics discovered by Hamilton (see, for instance, Lanczos [8]; Rund [11]). We shall find it more useful and suggestive to work in phase space since that makes the action of canonical transformations easier to visualise.

We consider a classical dynamical system with $n$ degrees of freedom and a $2 n$ dimensional phase space with canonical coordinates $q_{r}, p_{r}, r=1,2, \ldots, n$. The same system of coordinates will be used throughout the discussion, so that canonical transformations will always be interpreted in the active sense as arising from invertible mappings of the phase space onto itself. The condition for such a mapping to be canonical is given in the appendix (eq. (A.1)).

Let $M$ be an $m$-dimensional hypersurface embedded in the $2 n$ dimensional phase space, and let the real independent variables $u_{\alpha}, a=1,2, \ldots, m$, give a parametrisation of $M$. The points of $M$ then appear as $q_{r}(u), p_{r}(u)$. The Lagrange brackets (LB) of the $u_{\alpha}$ with one another are defined as

$$
\left[u_{\alpha}, u_{\beta}\right]=\frac{\partial q_{r}(u)}{\partial u_{\alpha}} \frac{\partial p_{r}(u)}{\partial u_{\beta}}-\frac{\partial q_{r}(u)}{\partial u_{\beta}} \frac{\partial p_{r}(u)}{\partial u_{\alpha}}
$$

[Summation over repeated indices is understood]. With these LB's as elements we can set up an $m \times m$ real antisymmetric matrix. We assume for simplicity that this matrix has constant rank over $M$, and call it the symplectic rank of $M$. It is easily verified that the symplectic rank is independent of the choice of parameters $u_{\alpha}$. Let a canonical transformation now be given. Applied to the points of $M$ it leads to another $m$-dimensional hypersurface, $M^{\prime}$ say, for which the $u_{\alpha}$ again serve as parameters. The point $q_{r}(u), p_{r}(u)$ on $M$ is carried by the canonical transformation to $Q_{r}(u), P_{r}(u)$ on $M^{\prime}$. It now follows from the invariance of LB's under canonical transformations (proved in the appendix) that $M^{\prime}$ has the same symplectic rank as $M$. Thus the symplectic rank of a hypersurface in phase space is an intrinsic canonical invariant property of the hypersurface.

Let us now consider the case that $M$ has zero symplectic rank. The appendix shows that the functions $q_{r}(u), p_{r}(u)$ must then be such that we have

$$
p_{r}(u) \frac{\partial q_{r}(u)}{\partial u_{a}}=\frac{\partial s(u)}{\partial u_{\alpha}}
$$

for some function $s(u)$. If $m<n$, knowledge of $q_{r}(u)$ and $s(u)$ does not suffice to determine $p_{r}(u)$. Let us assume next that $m=n$ and that the $u_{\alpha}$ can be taken to be 
the position variables $q_{r}$. In that case we can set $s(q)$ in place of $s(u)$ in eq. (2) and see that on $M$ the momenta $p_{r}$ are functions of the $q_{r}$ :

$$
p_{r}=\frac{\partial s(q)}{\partial q_{r}}
$$

Thus an $n$-dimensional hypersurface of vanishing symplectic rank over which the $q_{r}$ are independent variables is uniquely determined by any given function $s(q)$ on configuration space; and conversely such a hypersurface determines an $s(q)$ up to an additive constant. We shall use the notation $\Gamma[s(q)]$ for such hypersurfaces in phase space, additional arguments of $s$ being inserted when necessary. Occasionally the arguments of $s$ will be omitted if no confusion is likely to arise.

Let $f(q, p)$ be a given phase space function. On restricting the arguments of $f$ to a $\Gamma[s]$ we produce a function on configuration space; this is achieved by substituting for $p_{r}$ in $f$ from eq. (3). We shall describe this process by which one passes from a phase space function to a configuration space function, given an $s(q)$, with this notation:

$$
f\left(q, \frac{\partial s(q)}{\partial q}\right) \equiv(f(q, p))_{\mathrm{s}}
$$

It is useful to know the relationship between the PB operation and this restriction operation. If $g(q, p)$ is another phase space function, the $\mathrm{PB}$ of $f$ with $g$ is a third phase space function given by

$$
\{f, g\}=\frac{\partial f}{\partial q_{r}} \frac{\partial g}{\partial p_{r}}-\frac{\partial f}{\partial p_{r}} \frac{\partial g}{\partial q_{r}}
$$

Naturally the restriction of $\{f, g\}$ to $\Gamma[s]$ cannot be expressed in terms of the restrictions of $f$ and $g$ alone; however with the help of eq. (3) one easily finds the very important result

$$
(\{f, g\})_{s}=\frac{\partial(f)_{s}}{\partial q_{r}}\left(\frac{\partial g}{\partial p_{r}}\right)_{s}-\left(\frac{\partial f}{\partial p_{r}}\right)_{s} \frac{\partial(g)_{s}}{\partial q_{r}}
$$

We will use this formula repeatedly in what follows.

With the concepts introduced so far, let us examine the action of canonical transformations on hypersurfaces of the type $\Gamma[s]$. We shall only be interested in infinitesimal transformations generated by given functions $A(q, p)$ on phase space. The explicit formula for such canonical transformations is given in eq. (A.2) of the appendix. A concise notation for the infinite power series occurring in the transformation is achieved if we introduce a partial differential operator $A^{*}$ associated with the function $A$ (the asterisk is always used in this sense throughout the paper, and never to denote complex conjugation):

$$
A^{*}=\frac{\partial A}{\partial q_{r}} \frac{\partial}{\partial p_{r}}-\frac{\partial A}{\partial p_{r}} \frac{\partial}{\partial q_{r}} \cdots
$$


The action of $A^{*}$ on a function $B(q, p)$ then produces the $\mathrm{PB}\{A, B\}$. Then, the canonical transformation with parameter $\epsilon$ and infinitesimal generator $A(q, p)$ takes the form

$$
Q_{r}(q, p, \epsilon)=\exp \left(\epsilon A^{*}\right) q_{r}, P_{r}(q, p, \epsilon)=\exp \left(\epsilon A^{*}\right) p_{r}
$$

The transformation itself may be denoted by the symbol exp $\left(\epsilon A^{*}\right)$. Let us now apply this canonical transformation to the points comprising a given hypersurface $\Gamma[s]$. Since we shall be interested only in terms up to at most second order in the (small) parameter $\epsilon$, we may assume that the image of $\Gamma[s]$ also permits the use of the $q_{r}$ as independent parameters over it; thus this image must be $\Gamma\left[s^{\prime}\right]$ for some $s^{\prime}(q)$. We can in principle get $s^{\prime}$ using eq. (A.3) of the appendix: if $Q_{r}, P_{r}$ is the image in $\Gamma\left[s^{\prime}\right]$ of $q_{r}, p_{r}$ in $\Gamma[s]$, then

$$
P_{r} d Q_{r}=d\left[s(q)+\epsilon\left(A-p_{r}\left\{q_{r}, A\right\}\right)_{s}-\frac{\epsilon^{2}}{2}\left(\left\{A, p_{r}\left\{q_{r}, A\right\}\right\}\right)_{s} \cdots\right]
$$

By expressing the function within square brackets as a function of $Q$ rather than $q$, we discover the functional form of $s^{\prime}$. After some algebra we get the result

$$
s^{\prime}(q)=s(q)+\epsilon(A)_{s}+\frac{\epsilon^{2}}{2} \frac{\partial(A)_{s}}{\partial q_{r}}\left(\frac{\partial A}{\partial p_{r}}\right)_{s} \ldots
$$

(One recognises that this is just the solution of the H-J equation corresponding to $A$ and with $\epsilon$ as parameter). So we can also write

$$
\begin{aligned}
& \exp \left(-\epsilon A^{*}\right) \Gamma[s]= \\
& \quad \Gamma\left[s+\epsilon(A)_{s}+\frac{\epsilon^{2}}{2} \frac{\partial(A)_{s}}{\partial q_{r}}\left(\frac{\partial A}{\partial p_{r}}\right)_{s} \cdots\right]
\end{aligned}
$$

The negative sign in the exponent is introduced on purpose with a view to securing the Baker-Campbell-Hausdorff formula in its usual form later on. We must note two important points in this connection. The first is that whereas in eq. (8) $A^{*}$ is to be interpreted as a partial differential operator that acts on any function of $q, p$ that may stand to its right, in eq. (11) it is not to be interpreted literally in this sense but only symbolically. In fact the action of the symbol $\exp \left(-\epsilon A^{*}\right)$ on a hypersurface $\Gamma$ is completely defined by eq. (11). The second is that eq. (11) does not tell us how each point on $\Gamma[s]$ is affected by the canonical transformation but only how the function $s$ is affected; thus it only specifies the image of $\Gamma[s]$ as a whole.

In the remainder of this section we use the definitions and results obtained thus far to discuss some of the kinematic features associated with the H-J form of dynamics. It will be seen that these are very similar to well known features of quantum mechanics. The H-J equation per se is taken up in the succeeding sections. Let us ask first under what conditions a hypersurface $\Gamma[s]$ is invariant under a canonical transformation $\exp \left(-\epsilon A^{*}\right)$. Working only to the order $\epsilon$, we see from eq. (11), that this happens if and only if

$$
(A)_{s} \equiv A\left(q, \frac{\partial s}{\partial q}\right)=a,
$$


where $a$ is some constant. This equation can be called the H-J equation (timeindependent!) corresponding to the function $A(q, p)$. Thus $\Gamma[s]$ is invariant under $\exp \left(-\epsilon A^{*}\right)$ if and only if $s$ obeys the H-J equation corresponding to $A$ for some value of the constant $a$. This can be taken to be the analogue of the following statement in quantum mechanics: A (pure) physical state is unaffected by the unitary transformation generated by a given hermitian operator if and only if the corresponding state vector is an eigenvector of the operator in question. The fact that adding a constant to $s$ does not change $\Gamma[s]$ corresponds exactly to the fact that a physical state is unaltered when the state vector is multiplied by a phase factor. [Incidentally when eq. (12) is obeyed the $\epsilon^{2}$ term in eq. (11) is identically zero; this will persist in higher orders]. When eq. (12) holds, the structure of $\Gamma[s]$ can be described in this way: Clearly the orbits of points in $\Gamma[s]$ under the one-parameter family (group) of canonical transformations $\exp \left(\epsilon A^{*}\right)$ lie entirely in $\Gamma[s]$. Thus $\Gamma[s]$ can be reconstructed if one is given a suitable $(n-1)$-dimensional subsurface of it, a cross-section of the orbits, to all points of which one applies the canonical transformation $\exp \left(\epsilon A^{*}\right)$ for all real $\epsilon$. This is just the way in which solutions of the time-independent $\mathrm{H}-\mathrm{J}$ equation can be generated, as described in the appendix.

Suppose next that $\Gamma[s]$ is invariant under the canonical transformations generated by $A(q, p)$ as well as under those generated by some $B(q, p)$. Thus $s$ must obey two H-J equations simultaneously:

$$
(A)_{s}=a,(B)_{s}=b
$$

Using this in the PB formula eq. (6) we see immediately that

$$
(\{A, B\})_{s}=0
$$

Thus $s$ obeys the H-J equation corresponding to the phase space function $\{A, B\}$ with the constant on the right being zero. (This is a known result; see for instance Caratheodory [3] $\S 58$ ). This is the analogue to the statement in quantum mechanics that if a state vector is simultaneously an eigenvector of two distinct operators, then it is also an eigenvector of their commutator with eigenvalue zero. Conversely, if the PB $\{A, B\}$ is non zero throughout phase space, which happens if $A$ and $B$ form a canonically conjugate pair of functions, no function $s(q)$ can obey simultaneously the $\mathrm{H}-\mathrm{J}$ equations corresponding to $A$ and to $B$.

\section{Symmetry transformations and the $\mathbf{H}-\mathrm{J}$ equation}

We analyse in this section the action of symmetries of a dynamical system on the solutions of the H-J equation. For this purpose we first build up a geometrical picture for the solutions themselves. Let the possibly explicitly time-dependent Hamiltonian be $H(q, p, t)$ and let $S(q ; t)$ be a solution of the H-J equation

$$
(H(q, p, t))_{S}+\frac{\partial S(q ; t)}{\partial t}=0
$$


For each time $t$ we construct the corresponding hypersurface $\Gamma[S(q ; t)]$ in phase space. This hypersurface then moves with time, always preserving its character of being $n$-dimensional and of having vanishing symplectic rank. That $S$ obeys eq. (15) can now be interpreted in this way: the hypersurface at time $t+\delta t$ coincides with the result of applying the infinitesimal canonical transformation with generator $-H(q, p, t) \delta t$ to the hypersurface at time $t$. This is entirely natural, but it is an interesting application of eq. (11). Retaining only terms up to first order in $\delta t$, we have:

$$
\begin{aligned}
\exp (\delta t & \left.H(q, p, t)^{*}\right) \Gamma[S(q ; t)] \\
& =\Gamma\left[S(q ; t)-\delta t(H(q, p, t))_{S}\right] \\
& =\Gamma\left[S(q ; t)+\delta t \frac{\partial S(q ; t)}{\partial t}\right] \\
& =\Gamma[S(q ; t+\delta t)] .
\end{aligned}
$$

Use of eq. (11) was followed by use of eq. (15). It is true that motion according to Hamilton's equations from time $t$ to $t+\delta t$ is an infinitesimal canonical mapping of phase space onto itself but that is not the content of eq. (16). Rather we must view eq. (16) as expressing the following property of $S:$ If $S$ is any solution whatsoever of the H-J eq. (15), the evolution in time of $\Gamma[S(q ; t)]$ as determined by the explicit time dependence of $S$ coincides with the canonical evolution as determined by eq. (11) with $H(q, p, t)$ as generator. This interpretation is well known. Equation (16) gives a geometrical picture of it.

Next suppose a phase space function $G(q, p, t)$ is given, which happens to be a constant of motion:

$$
\{G, H\}+\frac{\partial G}{\partial t}=0
$$

In the transformation theory of dynamics, this is usually interpreted thus: under the (time-dependent) canonical transformation generated by $G$ the Hamiltonian preserves its functional form. We wish to connect this with the properties of solutions of the H-J equation. Let us recall first the analogous situation in quantum mechanics. Let a quantum system have a Hamiltonian operator $\hat{H}(t)$ and let $\hat{G}(t)$ be a hermitian constant of motion, either or both possessing explicit time dependence (we work in the Schrödinger picture of quantum mechanics):

$$
-i[\hat{G}(t), \hat{H}(t)]+\frac{\partial \hat{G}(t)}{\partial t}=0 .
$$

If $|\psi(t)\rangle$ is any solution of the Schrödinger equation

$$
i \frac{d}{d t}|\psi(t)\rangle=\hat{H}(t)|\psi(t)\rangle,
$$


and one defines a unitary operator $U(\epsilon ; t)$ by

$$
U(\epsilon ; t)=\exp (i \in \hat{G}(t)),
$$

then one finds that

$$
\left|\psi^{\prime}(t)\right\rangle=U(\epsilon ; t)|\psi(t)\rangle
$$

is also a solution of the Schrödinger equation. It is very easy to show this for infinitesimal $\epsilon$, and with a little operator algebra for any $\epsilon$. Thus the (possibly time dependent) unitary transformation generated by a (possibly explicitly time dependent) constant of motion $\hat{G}$ maps any possible state of motion onto another such. We seek the corresponding result within H-J theory. Given $G(q, p, t)$ obeying eq. (17) and any solution $S(q ; t)$ of eq. (15), let us set up the time dependent hypersurface $\Gamma[S(q ; t)]$ and apply to it an infinitesimal canonical transformation with parameter $\epsilon$ and generator $G$ taken at time $t$; from eq. (11) we get

$$
\begin{aligned}
& \exp \left(-\epsilon G(q, p, t)^{*}\right) \Gamma[S(q ; t)]=\Gamma\left[S^{\prime}(q ; t)\right] \\
& S^{\prime}(q ; t)=S(q ; t)+\epsilon(G(q, p, t))_{S^{\prime}}
\end{aligned}
$$

We first confirm by direct calculation that $S^{\prime}$ too obeys the H-J equation, and then show it geometrically. The time arguments being $t$ throughout, we have:

$$
\begin{aligned}
(H)_{S^{\prime}}-(H)_{S} & =\epsilon\left(\frac{\partial H}{\partial p_{r}}\right)_{S} \frac{\partial(G)_{S}}{\partial q_{r}} \\
\frac{\partial S^{\prime}}{\partial t}-\frac{\partial S}{\partial t} & =\epsilon \frac{\partial(G)_{S}}{\partial t} \\
& =\epsilon\left(\frac{\partial G}{\partial t}\right)_{S}+\epsilon\left(\frac{\partial G}{\partial p_{r}}\right)_{S} \frac{\partial^{2} S}{\partial q_{r} \partial t} \\
& =\epsilon\left(\frac{\partial G}{\partial t}\right)_{S}-\epsilon\left(\frac{\partial G}{\partial p_{r}}\right)_{S} \frac{\partial(H)_{S}}{\partial q_{r}}
\end{aligned}
$$

By combining these with the PB restriction formula (6) and with the restriction of eq. (17) to $\Gamma[S(q ; t)]$ we see immediately that $S^{\prime}(q ; t)$ is also a solution of the H-J equation. Thus we have the classical result that any constant of motion $G$ brings with it a one-to-one mapping of the manifold of solutions of the H-J equation onto itself. Recalling that, as shown in the appendix, each solution $S(q ; t)$ of the H-J equation is determined in a one-one manner by a function $s(q)$ on configuration space, we can also say: the phase space canonical transformation generated by $G$ gives rise to a mapping of configuration space functions onto themselves that preserves the H-J equation.

It is illuminating to give another, more geometrical, derivation of this result. This is where we need to use the second order terms in $\epsilon$ appearing on the right hand 
side of eq. (11). Let $\epsilon, \epsilon^{\prime}$ be two independent small parameters, $A(q, p)$ and $B(q, p)$ two phase space functions, and $\Gamma[s]$ a given hypersurface. We wish to calculate the result of applying two infinitesimal canonical transformations in succession to $\Gamma[s]$. Let us write

$$
\exp \left(-\epsilon^{\prime} B^{*}\right) \exp \left(-\epsilon A^{*}\right) \Gamma[s]=\exp \left(-\epsilon^{\prime} B^{*}\right) \Gamma\left[s_{1}\right]=\Gamma\left[s_{2}\right]
$$

Our objective is to relate $s_{2}$ to $s$ in the manner of eq. (11) via a single canonical transformation, retaining at all stages terms that are at most of second order in $\epsilon$ and $\epsilon^{\prime}$ jointly. We relate $s_{1}$ to $s$, and then $s_{2}$ to $s_{1}$, and retain the terms of interest to get:

$$
\begin{aligned}
s_{1}= & s+\epsilon(A)_{s}+\frac{\epsilon^{2}}{2} \frac{\partial(A)_{s}}{\partial q_{r}}\left(\frac{\partial A}{\partial p_{r}}\right)_{s} ; \\
s_{2}= & s_{1}+\epsilon^{\prime}(B)_{s_{1}}+\frac{\epsilon^{\prime 2}}{2} \frac{\partial(B)_{s_{1}}}{\partial q_{r}}\left(\frac{\partial B}{\partial p_{r}}\right)_{s_{1}} \\
& \simeq s+\epsilon(A)_{s}+\frac{\epsilon^{2}}{2} \frac{\partial(A)_{s}}{\partial q_{r}}\left(\frac{\partial A}{\partial p_{r}}\right)_{s}+\epsilon^{\prime}\left[(B)_{s}+\epsilon\left(\frac{\partial B}{\partial p_{r}}\right)_{s} \frac{\partial(A)_{s}}{\partial q_{r}}\right] \\
& +\frac{\epsilon^{\prime 2}}{2} \frac{\partial(B)_{s}}{\partial q_{r}}\left(\frac{\partial B}{\partial p_{r}}\right)_{s} \\
& =s+\left(\epsilon A+\epsilon^{\prime} B\right)_{s}+\frac{1}{2} \frac{\partial\left(\epsilon A+\epsilon^{\prime} B\right)_{s}}{\partial q_{r}}\left(\frac{\partial\left(\epsilon A+\epsilon^{\prime} B\right)}{\partial p_{r}}\right)_{s} \\
& +\frac{1}{2} \epsilon \epsilon^{\prime}\left[\frac{\partial(A)_{s}}{\partial q_{r}}\left(\frac{\partial B}{\partial p_{r}}\right)_{s}-\left(\frac{\partial A}{\partial p_{r}}\right)_{s} \frac{\partial(B)_{s}}{\partial q_{r}}\right] \\
& \approx s+(C)_{s}+\frac{1}{2} \frac{\partial(C)_{s}}{\partial q_{r}}\left(\frac{\partial C}{\partial p_{r}}\right)_{s}, \\
C= & \epsilon A+\epsilon^{\prime} B+\frac{1}{2} \epsilon \epsilon^{\prime}\{A, B\} .
\end{aligned}
$$

Note the use of eq. (6) at the last step but one in the above. We thus have the formula, valid to quadratic terms,

$$
\begin{aligned}
& \exp \left(-\epsilon^{\prime} B^{*}\right) \exp \left(-\epsilon A^{*}\right) \Gamma[s] \\
& \quad=\exp \left(-\epsilon A^{*}-\epsilon^{\prime} B^{*}-\frac{1}{2} \epsilon \epsilon^{\prime}\{A, B\}^{*}\right) \Gamma[s]
\end{aligned}
$$

This is the Baker-Campbell-Hausdorff formula for composition of canonical transformations, to lowest nontrivial order. (For more details see, for instance, Sudarshan and Mukunda [16]). On interchanging the roles of $A$ and $B$, we get a similar formula; and comparing the two we get, again to the second order, the result

$$
\begin{aligned}
& \exp \left(-\epsilon^{\prime} B^{*}\right) \exp \left(-\epsilon A^{*}\right)=\exp \left(-\epsilon \epsilon^{\prime}\{A, B\}^{*}\right) \exp \left(-\epsilon A^{*}\right) \exp \left(-\epsilon^{\prime} B^{*}\right) \\
& \simeq \exp \left(-\epsilon\left(A+\epsilon^{\prime}\{A, B\}\right)^{*}\right) \exp \left(-\epsilon^{\prime} B^{*}\right)
\end{aligned}
$$


valid in the sense of eq. (11) when both sides are applied to any $\Gamma[s] . \quad$ In this general result, let us take $A$ to be the constant of motion $G(q, p, t), B$ the Hamiltonian $H(q, p, t)$ and $-\epsilon^{\prime}$ an infinitesimal time interval $\delta t$. Because of eq. (17) the combination of $A$ and $B$ on the right hand side of eq. (25) becomes

$$
A+\epsilon^{\prime}\{A, B\}=G(q, p, t+\delta t)
$$

If we now apply both sides of eq. (25) to a hypersurface $\Gamma[S(q, t)]$ where $S$ is any solution of the H-J eq. (15) and use eq. (16) as well as the definition of $S^{\prime}$ given in eq. (20), we get:

$$
\begin{aligned}
\exp \left(\delta t H(q, p, t)^{*}\right) \Gamma\left[S^{\prime}(q ; t)\right] \\
\quad=\exp \left(-\epsilon G(q, p, t+\delta t)^{*}\right) \Gamma[S(q ; t+\delta t)] \\
\quad=\Gamma\left[S^{\prime}(q ; t+\delta t)\right]
\end{aligned}
$$

Thus we have recovered the result that $S^{\prime}$ obeys the H-J equation if $S$ does.

A well known feature of classical (and quantum) mechanics is the dual role played by every dynamical variable. On the one hand it assumes a value (expectation value) in a given state of motion, which value does not change with time if the variable is a constant of motion. On the other hand, the variable acts as the generator of canonical (unitary) transformations. These two roles come across particularly clearly in the above analysis showing how a constant of motion acts on a solution of the $\mathrm{H}-\mathrm{J}$ equation to produce another solution. Each solution $S(q ; t)$ of the H-J equation describes a family of solutions of the equations of motion corresponding to all initial phases lying on $\Gamma[s]$ where $s$ is the value of $S$ at $t=0$. A constant of motion $G(q, p, t)$ has a time-independent value on each one of the trajectories in the family but the value of $G$ will generally vary over the family. This variation is present when the restriction of $G(q, p, 0)$ to $\Gamma[s]$ is a non constant function of $q$ : and exactly in that case the new solution $S^{\prime}$ to the $H-J$ equation obtained from $S$ through. eq. (20) differs from $S$ and describes a different family. But if $(G(q, p, 0))_{s}$ is independent of $q$, then the value of $G$ is constant over the family at $t=0$ and so remains constant for all $t$. Formally we see from eqs (21b) and (17) that, the time arguments being $t$ throughout,

$$
\frac{\partial(G)_{S}}{\partial t}=-\left(\frac{\partial H}{\partial p_{r}}\right)_{S} \frac{\partial(G)_{S}}{\partial q_{r}}
$$

so that as is to be expected

$$
(G(q, p, 0))_{s}=\text { constant }=g \Rightarrow(G(q, p, t))_{S}=g .
$$

For such a solution $S$ of the H-J equation the new solution $S^{\prime}$ is essentially the same as $S$, meaning that the transformation generated by $G$ has no effect on $S$ at all.

For the sake of complete generality we have treated the situation where both the Hamiltonian and the constant of motion were permitted to have explicit time dependences. The simpler statements valid if these dependences are absent are easily obtained from the results given already. 


\section{The time-independent $H-J$ equation}

We now consider conservative dynamical systems and the role of the time independent H-J equation. The general method by which solutions to this equation arise is explained in the appendix. We are particularly interested in the relationship between solutions of the time dependent and independent $\mathrm{H}-\mathrm{J}$ equations and the points of similarity to the situation in quantum mechanics. We recall the latter situation first. Suppose $|\psi(t)\rangle$ is a general solution of the Schrödinger eq. (19) with a time-independent Hamiltonian operator $\hat{H}$, determined by the initial state $|\psi\rangle$ at $t=0$ :

$$
|\psi(t)\rangle=\exp (-i t \hat{H})|\psi\rangle
$$

This solution can be expanded linearly in terms of energy eigenstates with harmonic time dependence, say:

$$
|\psi(t)\rangle=\int d E|\phi(E)\rangle \exp (-i E t)
$$

For simplicity we have assumed that $\hat{H}$ has a continuous spectrum, and $|\phi(E)\rangle$ is a (not necessarily normalised) eigenvector of $\hat{H}$ with eigenvalue $E$. The values of $E$ that occur, and the corresponding vectors $|\phi(E)\rangle$, are all characteristic of the state at time $t=0$ :

$$
|\psi\rangle=\int d E|\phi(E)\rangle
$$

The rule by which each of the 'pieces' $|\phi(E)\rangle$ present in $|\psi\rangle$ can be projected out of $|\psi\rangle$ reads:

$$
|\phi(E)\rangle=\frac{1}{2 \pi} \int_{-\infty}^{\infty} d t \exp (i E t) \exp (-i t \hat{H})|\psi\rangle=\delta(E-\hat{H})|\psi\rangle
$$

In words: One subjects $|\psi\rangle$ to time translation by all possible amounts, multiplies by the factor $\exp (i E t)$, and integrates over all time. By this method one produces, from any given $|\psi\rangle$, energy eigenstates for all those values of $E$ that are 'present' in $|\psi\rangle$.

There is an analogous procedure in the classical H-J theory (see, for instance, Sommerfeld [15]). Let $S(q ; t)$ be a nonlinearly time dependent solution of the H-J equation

$$
(H(q, p))_{s}+\frac{\partial S(q ; t)}{\partial t}=0
$$

If we introduce an energy parameter $E$ by

$$
(H(q, p))_{s}=E,
$$


we regard $E$ as a function of $q$ and $t$, and conversely by inversion $t$ as a function of $q$ and $E$ :

$$
t=a(q ; E)
$$

We now eliminate the variable $t$ in favour of $E$ by a Legendre transformation and define a function $W(q ; E)$ by

$$
\begin{aligned}
W(q ; E) & =S(q ; t)+E t \\
& =S(q ; a(q ; E))+E a(q ; E) .
\end{aligned}
$$

One sees then using eq. (34) that

$$
\frac{\partial W(q ; E)}{\partial q_{r}}=\left(\frac{\partial S(q ; t)}{\partial q_{r}}\right)_{t=a} .
$$

Of course the differentiation of $W$ is at constant $E$, while that of $S$ is at constant $t$ followed by substitution for $t$. Equations (35) and (38) now show that $W(q ; E)$ is a solution of the time independent $\mathrm{H}-\mathrm{J}$ equation:

$$
(H(q ; p))_{W}=E
$$

Analysing this construction more closely we see the following. We know that a solution $S(q ; t)$ of eq. (34) arises uniquely from an arbitrarily specified function $s(q)$ on configuration space to which $S$ must reduce at $t=0$. On the other hand, the solution $W(q ; E)$ to eq. (39) is determined uniquely given $S$ and $E$. It follows that every function $s(q)$ on configuration space must lead in a definite manner to definite solutions $W(q ; E)$ of eq. (39) for each $E$ in a certain range. It is natural to trace the emergence of $W(q ; E)$ directly from $s(q)$, analogously to the quantum result (33). It is possible to do this using the geometrical constructs of section 1 and the results given in the appendix. Given $s(q)$ we first set up the $n$-dimensional hypersurface $\Gamma[s]$. As $q$ runs over all of configuration space the restriction $(H)_{s}$ of the Hamiltonian to $\Gamma[s]$ takes up a certain range of values. For any $E$ in this range we define an (n-1)-dimensional region in configuration space by the equation

$$
(H(q, p))_{s}=E \text {. }
$$

Suppose $u_{a}, a=1,2, \ldots, n-1$, give a parametrisation of this region, so that functions $q_{r}(u)$ solve eq. (40). The restriction of the $q_{r}$ to this region of configuration space gives a sub surface in $\Gamma[s]$ as well, of dimension $(n-1)$. The values of the momenta in this subsurface could be expressed as $\partial s(q) / \partial q_{r}$ with the $q_{r}$ being restricted after the partial differentiations are done. Alternatively we can observe the restriction (40) on the $q_{r}$ from the start by saying that the momenta are determined in the region considered by the equations

$$
\begin{gathered}
p_{r}(u) \frac{\partial q_{r}(u)}{\partial u_{\alpha}}=\frac{\partial s^{\prime}(u)}{\partial u_{\alpha}} \\
H(q(u), p(u))=E,
\end{gathered}
$$


where

$$
s^{\prime}(u)=s(q(u))
$$

(For ease in writing the additional argument $E$ has been omitted in both $q(u)$ and $p(u)$ ). Equation (41) are of just the form of eq. (A.16), so from $s(q)$ and $E$ we have produced an $(n-1)$ parameter family of initial phases $q_{r}(u), p_{r}(u)$. From the appendix we know that these are just the ingredients needed for the construction of a solution of the time independent H-J equation with energy $E$ : we must apply all possible time translations to this family of initial phases, thereby generating an $n$-dimensional hypersurface $\Gamma_{E}$ in phase space which is guaranteed to be $\Gamma[W(q ; E)]$ for some $W(q ; E)$ obeying eq. (39).

To explicitly find $W(q ; E)$ we must use $q_{r}$ as parameters over $\Gamma_{E}$ and express the momenta $p_{r}$ at points on $\Gamma_{E}$ in the form

$$
p_{r}=\frac{\partial W(q ; E)}{\partial q_{r}}
$$

Figure 1 clarifies the construction: The $n$-dimensional hypersurface $\Gamma[s]$ appears as a 'line '; its ( $n-1)$-dimensional subsurface defined by eq. (40) appears as a 'point '; the application of all possible time translations to this 'point' generates the ( $n$ dimensional) 'line' $\Gamma_{E}$. A point on $\Gamma_{E}$ with configuration $q$ determines a corresponding momentum $p$. This phase space point $(q, p)$ lies on the hypersurface $\Gamma[S(q ; t)]$, obtained by canonical transformation from $\Gamma[s]$, for some $t$. The figure shows that $t$ is determined in terms of $q$ and $E$ just by eqs (35) and (36)-thus clarifying the meaning of turning time into a function of $q$ and $E$. If the $q_{r}$ are viewed as parametrising $\Gamma[S(q ; t)], p_{r}$ are given by

$$
p_{r}=\frac{\partial S(q ; t)}{\partial q_{r}}
$$

This is ' differentiation at constant $t$ '. We want to express the same $p_{r}$ in the manner of eq. (43) which is ' differentiation at constant $E$ ' corresponding to the use of the

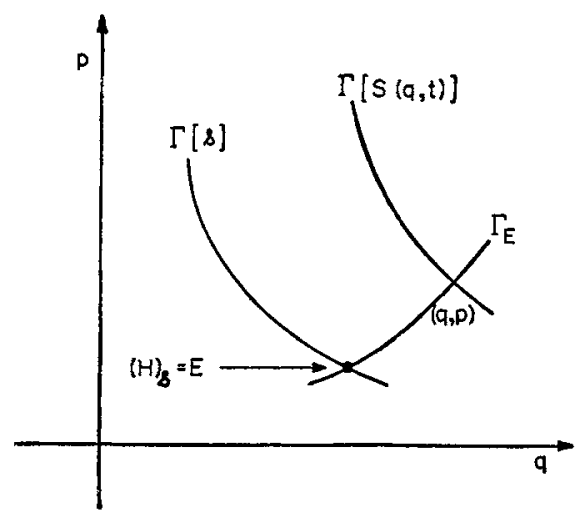

Figure 1. Generation of solutions to time-independent H-J equation. 
$q_{r}$ as parameters for $\Gamma_{E}$. This means that we must use eq. (35) to eliminate $t$ in favour of $E$, and the Legendre transformation (37) gives just the correct $W$ in terms of which we secure eq. (43).

Thus the generation of solutions $W(q ; E)$ to the time-independent $\mathrm{H}-\mathrm{J}$ equation starting from any configuration space function $s(q)$ is understood and seen to be similar in several respects to the situation in quantum mechanics.

\section{H-J equations on a Lie group}

Though it is not directly related to the topics discussed in the preceding sections, we describe very briefly in this section an extension of the H-J theory in which the single time parameter is replaced by an element of a Lie group. This is natural to consider for those dynamical systems which possess an invariance group larger than the group of time translations.

Let $a^{j}, j=1,2, \ldots, N$, be a coordinate system for an $N$-parameter Lie group $\mathscr{G}$, normalised so that $a^{j}=0$ at the identity. Let the product of the elements $a, \beta$ have coordinates $f^{j}(\alpha, \beta)$. The functions defined by

$$
\eta_{j}^{k}(\alpha)=\left(\frac{\partial f^{k}(\beta, \alpha)}{\partial \beta^{j}}\right)_{\beta=0}
$$

are known to form a nonsingular matrix reducing to the unit matrix at $a=0$; and obey

$$
\left[\eta_{j}^{u}(\alpha) \frac{\partial}{\partial a^{u}}, \eta_{k}{ }^{j}(a) \frac{\partial}{\partial a^{j}}\right]=-C_{j k}^{l} \eta_{l}^{w}(\alpha) \frac{\partial}{\partial a^{w}},
$$

where the $C^{\prime}$ s are the structure constants of $\mathscr{G}$. (For a concise account see, for instance, Sudarshan and Mukunda [16] Ch. 13).

A Hamiltonian dynamical system with invariance group $\mathscr{G}$ supplies us with a set of phase space functions $G_{j}(q, p)$ giving a PB realisation of the Lie algebra of $\mathscr{G}$ (Saletan and Cromer [12], Ch. IX; Sudarshan and Mukunda [16] Ch. 14):

$$
\left\{G_{j}(q, p), G_{k}(q, p)\right\}=C_{j_{k}}^{l} G_{l}(q, p)
$$

For simplicity the possible presence of neutral elements in the realisation is ignored. These relations ensure that the finite canonical transformarions generated by the $G_{j}(q, p)$ give a realisation of $\mathscr{G}$. If $\mathscr{G}$ consisted of time translations alone, we would have just one $G$, the Hamiltonian.

In this framework one sees that the single $\mathrm{H}-\mathrm{J}$ equation of ordinary dynamics gets enlarged to a system of $\mathrm{H}-\mathrm{J}$ equations, one for each $a^{j}$, in this form:

$$
\eta_{j}{ }^{k}(a) \frac{\partial S(q ; a)}{\partial a^{k}}+\left(G_{j}(q, p)\right)_{S}=0
$$

The unknown here is a configuration space function $S$ that depends also on the $N$ parameters $a^{j}$. Equations (46) and (47) jointly ensure the integrability of this system 
of $\mathrm{H}-\mathrm{J}$ equations, provided we use formula (6) as well. We do not analyse this system further here, but point it out as an interesting extension of the usual theory.

\section{Conclusion}

We have provided a general analysis of the Hamilton-Jacobi formulation of classical dynamics laying particular emphasis on the description of symmetries and invariance. We have tried to see to what extent the classical transformation theory adapts itself naturally to this form of dynamics. This study has been motivated by certain wellknown features of the Schrödinger form of quantum mechanics as well as the desire to understand better the grouping together of classical states of motion into families that is characteristic of the Hamilton-Jacobi method (cf. the remarks of Dirac [4]). Our interest has been to trace the connections existing between different elements of the theory and not to provide new techniques for solving known equations.

As an example of the better insight provided by this work into standard applications of the H-J theory, we mention the following. The cases where the H-J equation is explicitly solved are rather few in number, usually corresponding to situations where the method of separation of variables works. It is well known that these are the cases possessing specific symmetry properties; these symmetries lead to special coordinate systems in which the H-J equation separates. From sections 1 and 2 the significance of these cases is realised clearly and in a deeper way. These are just the situations wherein a maximal number of global constants of motion exist, with the property that any two of them are in involution, i.e., have vanishing PB. Precisely then does there exist a single function $S$ that simultaneously satisfies the $\mathrm{H}-\mathrm{J}$ equations corresponding to each of these constants of motion. In fact $S$ is (essentially) uniquely determined by these equations. This is exactly like finding simultaneous eigenvectors for a complete commuting set of operators in quantum mechanics.

The discussion in this paper has been restricted (implicitly at least!) to local properties in phase space; it is extremely hard to make statements about properties in the large. In spite of this we feel that the methods used here are interesting in their own right and may suggest new points of view in analysing the semiclassical regime of quantum mechanics and its relation to classical mechanics. We hope to have successfully brought out some of the beautiful aspects of the Hamilton-Jacobi form of dynamics so that it may be viewed as something more than merely a means to solving Hamilton's equations of motion.

\section{Appendix}

The material in this appendix is presented solely for the convenience of the reader, no specifically new results being involved. Several well-known ideas have been put together in a compact form especially suitable for the applications made in the body of the paper.

Let $q_{r}, p_{r}, r=1,2, \ldots, n$, be a canonical coordinate system for a $2 n$-dimensional phase space. There are many equivalent ways of defining a canonical transformation, of which we choose the one based on the differential expression $p_{r} d q_{r}$. An 
invertible one-to-one mapping of the phase space onto itself, taking a general point $q_{r}, p_{r}$ to the point $Q_{r}(q, p), P_{r}(q, p)$, is a canonical transformation if the expression

$$
P_{r} d Q_{r}-p_{r} d q_{r}
$$

is a perfect differential, i.e., if there exists a phase space function $w(p, q)$ such that

$$
P_{r} d Q_{r}-p_{r} d q_{r}=d w(q, p)
$$

Here, $d q_{r}$ and $d p_{r}$ may be pictured as independent small changes in the coordinates of the point $q_{r}, p_{r}$, leading via the mapping to definite small changes $d Q_{r}, d P_{r}$ in the coordinates of the image point $Q_{r}, P_{r}$. If the canonical transformation depends on one or more parameters, these will enter as arguments of $w$ as well.

A given function $A(q, p)$ on phase space can be used as the (infinitesimal) generator of a one-parameter family of canonical transformations in a well-known way. If $\epsilon$ is the (real) parameter of the transformation, the equations of the transformation can be formally written using PB notation as:

$$
\begin{aligned}
& Q_{r}(q, p, \epsilon)=q_{r}+\epsilon\left\{A, q_{r}\right\}+\frac{\epsilon^{2}}{2 !}\left\{A,\left\{A, q_{r}\right\}\right\}+\ldots, \\
& P_{r}(q, p, \epsilon)=p_{r}+\epsilon\left\{A, p_{r}\right\}+\frac{\epsilon^{2}}{2 !}\left\{A,\left\{A, p_{r}\right\}\right\}+\ldots
\end{aligned}
$$

Naturally, only canonical transformations continuously connected to the identity transformation can be produced in this way with the help of an infinitesimal generator. Putting eq. (A2) into eq. (A1), the function $w(q, p, \epsilon)$ can be calculated as a power series in $\epsilon$. Up to second order, the result is

$$
P_{r} d Q_{r}-p_{r} d q_{r}=d\left[\epsilon\left(A-p_{r}\left\{q_{r}, A\right\}\right)-\frac{\epsilon^{2}}{2}\left\{A, p_{r}\left\{q_{r}, A\right\}\right\} \ldots\right]
$$

While $q_{r}$ and $p_{r}$ appear as independent variables in eq. (A 1), one can obtain useful results by restricting them in various ways. Let $M$ be an $m$-dimensional hypersurface embedded in the $2 n$-dimensional phase space, and let $u_{\alpha}, \alpha=1,2, \ldots, m$, give a real parametrisation of $M$ in the sense that the coordinates of points of $M$ can be written as $q_{r}(u), p_{r}(u)$ with the $u_{a}$ being independently variable. The canonical transformation applied to the points of $M$ leads to another $m$-dimensional hypersurface, $M^{\prime}$ say, with the image of the point $q_{r}(u), p_{r}(u)$ on $M$ being the point $Q_{r}(u), P_{r}(u)$ on $M^{\prime}$; thus the $u_{a}$ serve as parameters for $M^{\prime}$ as well. We now restrict $d q_{r}, d p_{r}$ to be just those possible small changes in $q_{r}, p_{r}$ that can be produced by independent small changes in the $u_{\alpha}$, so that along with $q_{r}, p_{r}$, the point $q_{r}+d q_{r}, p_{r}+d p_{r}$ also belongs to $M$. The canonical nature of the transformation then leads to an equation of the form

$$
P_{r}(u) \frac{\partial Q_{r}(u)}{\partial u_{a}}-p_{r}(u) \frac{\partial q_{r}(u)}{\partial u_{a}}=\frac{\partial w(u)}{\partial u_{a}}
$$


From here we conclude that

$$
\frac{\partial}{\partial u_{\beta}}\left(P_{r} \frac{\partial Q_{r}}{\partial u_{\alpha}}\right)-\frac{\partial}{\partial u_{\alpha}}\left(P_{r} \frac{\partial Q_{r}}{\partial u_{\beta}}\right)=\frac{\partial}{\partial u_{\beta}}\left(p_{r} \frac{\partial q_{r}}{\partial u_{\alpha}}\right)-\frac{\partial}{\partial u_{\alpha}}\left(p_{r} \frac{\partial q_{r}}{\partial u_{\beta}}\right),
$$

which is in fact an explicit expression of the invariance of the LB's among the $u_{a}$ under a canonical transformation:

$$
\left[u_{a}, u_{\beta}\right]_{Q, P}=\left[u_{\alpha}, u_{\beta}\right]_{q, p}
$$

In the remainder of this appendix we summarise briefly how general solutions to the time dependent H-J equation for a general Hamiltonian $H(q, p, t)$ and to the time independent $\mathrm{H}-\mathrm{J}$ equation for a conservative Hamiltonian $H(q, p)$, can in principle be constructed (For detailed discussions see Caratheodory [3], Rund [11]). The key idea to be used is of course the fact that time evolution according to Hamilton's equations of motion,

$$
Q_{r}=\left\{Q_{r}, H(Q, P, t)\right\}, \dot{P}_{r}=\left\{P_{r}, H(Q, P, t)\right\}
$$

is the continuous unfolding of a canonical transformation. As preparation for both cases we consider first the following situation. Choose as above an $m$-dimensional hypersurface $M$ with parameters $u_{\alpha}$ and consider the $m$-fold infinity of phases $q_{r}(u)$, $p_{r}(u)$ at an initial time $t=0$. Each of these initial phases gives rise via eq. (A7) to a corresponding phase space trajectory, the phase at time $t$ on a general one of these trajectories being $Q_{r}(t ; u), P_{r}(t ; u)$. Equation (A6) now states that the LB's $\left[u_{a}, u_{\beta}\right]$ are independent of time. It follows that these LB's vanish for all time over the entire family of trajectories if they vanish everywhere on $M$, i.e., the hypersurface $M$ is such that for a suitable function $s(u)$ we have

$$
p_{r}(u) \frac{\partial q_{r}(u)}{\partial u_{a}}=\frac{\partial s(u)}{\partial u_{a}}
$$

Let these equations be fulfilled. Then we see that, for the $m$-parameter family of trajectories being considered, eq. (A4) guarantees the existence of a function $w(t ; u)$ such that

$$
P_{r}(t ; u) \frac{\partial Q_{r}(t ; u)}{\partial u_{a}}=\frac{\partial}{\partial u_{a}}(s(u)+w(t ; u))
$$

In this general discussion all values of $m \leqslant n$ are permitted. In particular if $m<n$, knowledge of $s(u)$ and $q_{r}(u)$ does not suffice to determine $p_{r}(u)$ completely via eq. (A8).

To arrive at general solutions of the time dependent $\mathrm{H}-\mathrm{J}$ equation for a general Hamiltonian $H(Q, P, t)$, we specialise the above discussion by taking $M$ to be of dimension $n, m=n$, and assuming that the $q_{r}$ can be chosen as the independent parameters $u_{a}$ on $M$. Then eq. (A8) does determine the momenta $p_{r}$ on $M$ as functions of the $q_{r}$,

$$
p_{r}=\frac{\partial s(q)}{\partial q_{r}},
$$


and $M$ becomes a hypersurface $\Gamma[s]$ of the type introduced in section 1, determined completely by a given function $s(q)$ on configuration space. The function $s(q)$ then produces, according to the previous paragraph, an $n$-parameter family of trajectories with the phase at time $t$ on a general member being $Q_{r}(t ; q), P_{r}(t ; q)$. We assume next for each $t$ that the $Q_{r}$ are independent functions of the $q_{r}$. In that case, eq. (A9) shows that there exists a function $S(Q ; t)$ such that at each time $t$ we have

$$
P_{r} d Q_{r}=d S(Q ; t) .
$$

The family of trajectories is therefore such that the momenta are always determined by the positions through

$$
P_{\mathrm{r}}=\frac{\partial S(Q ; t)}{\partial Q_{\mathrm{r}}}
$$

But the family has been obtained by integrating the equations of motion (A7) for a particular collection of initial phases, so eq. (A12) must be consistent with eq. (A7). This means, on taking the total time derivative of both sides of eq. (A12) and then using eqs (A 7) and (A 12) in the result,

$$
\left(-\frac{\partial H(Q, P, t)}{\partial Q_{r}}\right)_{s}=\frac{\partial^{2} S}{\partial Q_{r} \partial t}+\frac{\partial^{2} S}{\partial Q_{r} \partial Q_{s}}\left(\frac{\partial H(Q, P, t)}{\partial P_{s}}\right)_{s},
$$

i.e.,

$$
\frac{\partial}{\partial Q_{r}}\left[(H(Q, P, t))_{S}+\frac{\partial S}{\partial t}\right]=0 .
$$

Since eq.(A11) leaves $S$ undetermined up to the addition of a time-dependent constant, we see that $S$ may be taken to obey the H-J equation

$$
(H(Q, P, t))_{S}+\frac{\partial S}{\partial t}=0 .
$$

This still leaves $S$ undetermined up to the addition of a time-independent constant. This ambiguity is eliminated by comparing eq. (A11) with eq. (A10) and requiring that at $t=0$ we have

$$
S(Q ; 0)=s(Q) .
$$

In this way, each given function $s(Q)$ on configuration space leads to a corresponding uniquely determined solution of the time dependent H-J eq. (A14).

Finally suppose $H(Q, P)$ is a Hamiltonian with no explicit time dependence and we wish to build up solu ions to the corresponding time-independent $\mathrm{H}-\mathrm{J}$ equation. In the general discussion preceding eq. (A9) we take $m=n-1$ and choose the functions $q_{r}(u)$ in some way. Thus the initial configurations belong to an $(n-1)$-dimensional 
region of configuration space. To determine the initial momenta $p_{r}(u)$ we impose $n$ equations

$$
\begin{aligned}
& p_{r}(u) \frac{\partial q_{r}(u)}{\partial u_{\alpha}}=\frac{\partial s^{\prime}(u)}{\partial u_{\alpha}}, \\
& H(q(u), p(u))=E .
\end{aligned}
$$

Here $s^{\prime}(u)$ is some function of the $(n-1)$ parameters $u_{a}$ and $E$ an arbitrarily chosen energy. (We assume these are independent equations for the $p_{r}(u)$. and skip writing the more correct form $p(u ; E))$. This $(n-1)$ parameter family of initial phases produces an $(n-1)$ parameter family of trajectories filling out an $n$-dimensional region in phase space. The phase at time $t$ on one of these trajectories can be written $Q_{r}$ $(t ; u ; E), P_{r}(t ; u ; E)$ and eq. (A9) guarantees the existence of a $V(t ; u ; E)$ such that

$$
P_{r}(t ; u ; E) \frac{\partial Q_{r}(t ; u ; E)}{\partial u_{\alpha}}=\frac{\partial V(t ; u ; E)}{\partial u_{a}}
$$

Energy conservation and eq (A 16b) jointly give

$$
H(Q(t ; u ; E), P(t ; u ; E))=E .
$$

Now we must extend eq. (A17) to cover the partial derivatives with respect to $t$ also. For this we show first that since $H$ has no explicit time dependence the LB's $\left[t, u_{\alpha}\right]$ are conserved:

$$
\begin{aligned}
& \frac{\partial}{\partial t}\left(\left[t, u_{a}\right]_{Q, P}\right)=\frac{\partial}{\partial t}\left(\frac{\partial Q_{r}}{\partial t} \frac{\partial P_{r}}{\partial u_{a}}-\frac{\partial Q_{r}}{\partial u_{\alpha}} \frac{\partial P_{r}}{\partial t}\right) \\
& =\frac{\partial}{\partial t}\left(\frac{\partial H(Q, P)}{\partial P_{r}} \frac{\partial P_{r}}{\partial u_{\alpha}}+\frac{\partial H(Q, P)}{\partial Q_{r}} \frac{\partial Q_{r}}{\partial u_{a}}\right) \\
& =\frac{\partial}{\partial t} \frac{\partial}{\partial u_{a}} H(Q, P)=\frac{\partial}{\partial u_{a}} \frac{\partial}{\partial t} H(q(u), p(u))=0 .
\end{aligned}
$$

[The arguments $t, u, E$ of $Q, P$ were omitted for simplicity]. Here we used only the conservation of $H$ but not the further property that for all members of the family of trajectories considered $H$ has the same value $E$. If we do take account of this property then the same steps as in eq. (A 19) but with $\partial / \partial t$ omitted show that the LB's $\left[t, u_{a}\right]$ in fact have the constant values zero:

$$
\left[t, u_{a}\right]=\frac{\partial}{\partial u_{a}} H(q(u), p(u))=0
$$

We express this as

$$
\frac{\partial}{\partial u_{a}}\left(P_{r} \frac{\partial Q_{r}}{\partial t}\right)=\frac{\partial}{\partial t}\left(P_{r} \frac{\partial Q_{r}}{\partial u_{a}}\right),
$$


recall that $V(t ; u ; E)$ in eq. (A 17) is at this stage undetermined up to addition of a function of $t$ (and $E$ ) alone, and so conclude that $V$ may be chosen so that we can write

$$
P_{r} d Q_{r}=d V(t ; u ; E)
$$

with all $n$ quantities $t, u_{\mathrm{r}}$ capable of independent variation. As the last step we assume that $Q_{\mathrm{r}}(t ; u ; E)$ are $n$ independent functions of $t, u_{a}$; this means that the $Q_{r}$ can serve as independent parameters for the $n$-dimensional region in phase space filled out by the trajectories. If then $V(t ; u ; E)$ is written as $W(Q ; E)$ eq. (A 22) gives

$$
P_{r}=\frac{\partial W(Q ; E)}{\partial Q_{r}}
$$

and this coupled with eq.(A18) shows that $W$ is a solution of the time-independent H-J equation

$$
H\left(Q, \frac{\partial W}{\partial Q}\right)=E
$$

To summarise, each solution of this equation arises by choosing an $(n-1)$ parameter family of initial positions $q_{r}(u)$, a function $s^{\prime}(u)$, and an energy $E$ in such a way that the initial momenta can be obtained from eq. (A 16), and such that in the resulting family of trajectories $Q_{r}$ can replace $t, u_{a}$ as independent variables. The $n$-dimensional region of phase space filled out by the trajectories determined by this $(n-1)$ parameter family of initial phases forms the hypersurface $\Gamma[W(q ; E)]$. The construction just outlined is essentially the one given by Einstein [5], while the one described by Schrödinger [13] is a special case of this one.

\section{References}

[1] Born M 1927 The Mechanics of the Atom (London: G. Bell and Sons), Ch. 1

[2] Brillouin L 1926 Comptes Rendus 18324

[3] Caratheodory C 1965 Calculus of Variations and Partial Differential Equations of the First Order Part I (San Francisco, California: Holden-Day)

[4] Dirac P A M 1951 Can. J. Math. 31

[5] Einstein A 1917 Verh.d. Deut. Phys. Ges. 1982

[6] Epstein P 1916 Phys. Zeitschr. 17148

[7] Kramers H A 1926 Z. Phys. 39828

[8] Lanczos C 1949 The Variational Principles of Mechanics (Toronto: University of Toronto Press) Ch. VIII

[9] Noether E 1918 Nachr. Ges. Wiss. Gott. 37

[10] Nordheim L and Fues E 1927 Die Hamilton-Jacobische Theorie der Dynamik Geiger-Scheel Handbuch der Physik Vol. V (Berlin: Springer) pp. 91-130

[11] Rund H 1966 The Hamilton-Jacabi Theory in the Calculus of Variations (London: D. Van Nostrand Co.)

[12] Saletan E J and Cromer A H 1971 Theoretical Mechanics (New York: John Wiley) Ch. IX

[13] Schrodinger E 1926 Ann. Phys. 79 361, 489; reprinted in Ludwig G 1968 Wave Mechanics (Oxford: Pergamon Press) 
[14] Sommerfeld A 1916 Ann. Phys. 511

[15] Sommerfeld A 1934 Atomic Structure and Spectral Lines 3rd ed. (London: Methuen and Co.) Vol. $1 \S 6$

[16] Sudarshan E C G and Mukunda N 1974 Classical Dynamics-A Modern Perspective (New York: John Wiley)

[17] Wentzel G 1926 Z. Phys. 38518

[18] Whittaker E T 1927 A Treatise on the Analytical Dynamics of Particles and Rigid Bodies 3rd ed. (Cambridge: University Press) Ch. XI, XII

[19] Wilson W 1915 Phil. Mag. 29795 